\title{
Frontières
}

\section{La construction du risque chez des hypertendus considérés inobservants à leurs médicaments antihypertenseurs}

\author{
Michelle Proulx, Nicole Leduc, Louise Vandelac, Rémi Guibert et Jean-Pierre \\ Grégoire
}

Volume 16, numéro 1, automne 2003

Remède ou poison ?

URI : https://id.erudit.org/iderudit/1073761ar

DOI : https://doi.org/10.7202/1073761ar

Aller au sommaire du numéro

Éditeur(s)

Université du Québec à Montréal

ISSN

1180-3479 (imprimé)

1916-0976 (numérique)

Découvrir la revue

Citer cet article

Proulx, M., Leduc, N., Vandelac, L., Guibert, R. \& Grégoire, J.-P. (2003). La construction du risque chez des hypertendus considérés inobservants à leurs médicaments antihypertenseurs. Frontières, 16(1), 51-56.

https://doi.org/10.7202/1073761ar

\section{Résumé de l'article}

Cet article a pour but d'illustrer les perceptions du risque chez des individus considérés inobservants à leurs médicaments antihypertenseurs, puis d'analyser dans quelle mesure ces perceptions influent sur la prise irrégulière du médicament. Il met en évidence certains des enjeux relatifs au transfert des connaissances auprès des publics, auxquels les cliniciens sont appelés à porter un intérêt croissant. 


\section{Résumé}

Cet article a pour but d'illustrer les perceptions du risque chez des individus considérés inobservants à leurs médicaments antihypertenseurs, puis d'analyser dans quelle mesure ces perceptions influent sur la prise irrégulière du médicament. II met en évidence certains des enjeux relatifs au transfert des connaissances auprès des publics, auxquels les cliniciens sont appelés à porter un intérêt croissant.

Mots clés: hypertendu - médicament antihypertenseur-risque-mortalité.

\section{Abstract}

This article illustrates the perceptions of risk for individuals with low adherence to their hypertension medications. It analyzes how these perceptions influence the irregular taking of medication. It highlights a number of issues inherent in the transfer of knowledge with different publics, especially those that clinicians are being incited to pay particular attention.

Key words: hypertension - hypertension medication - risk-mortality.

\section{LA CONSTRUCTION DU RISQUE CHEZ DES HYPERTENDUS considérés inobservants à leurs médicaments antihypertenseurs}

\section{Michelle Proulx, Ph.D., \\ santé publique, option Promotion de la santé, Médecine sociale et préventive, Université de Montréal.}

Nicole Leduc, Ph.D.,

professeure agrégée, Groupe de recherche interdisciplinaire en santé, Département d'administration de la santé, Université de Montréal.

Louise Vandelac, Ph.D., professeure titulaire, Département de sociologie, Université du Québec à Montréal.

Rémi Guibert, M.D., Ph.D., Groupe de recherche interdisciplinaire en santé

Jean-Pierre Grégoire, Ph.D., professeur titulaire, Faculté de pharmacie, Université Laval.

L'accent qui est actuellement mis sur le transfert des connaissances a souvent pour effet de submerger les publics d'informations sur le risque pour la santé, alors que la pertinence de ces informations et leurs possibilités d'application ne sont pas toujours évidentes aux yeux des individus concernés. Si la médecine a réussi à établir de façon relativement claire les niveaux de morbidité et de mortalité associés à certaines pathologies, néanmoins dans l'esprit des personnes aux prises avec un problème de santé, il en est souvent tout autrement. D'abord, bon nombre de personnes éprouvant des problèmes de santé sont à même de constater à quel point leurs expériences personnelles et celles observées dans leur entourage peuvent parfois défier les prédictions qui sont faites par les experts (Hunt et Emslie, 2001). Ajoutons que l'enchevêtrement des différentes rationalités et la concurrence des différentes manières de voir provenant de sources officielles et médiatiques ont également pour effet de miner leur confiance (Lupton, 1995).

Dans ce contexte, il est facile de comprendre que des individus puissent hésiter à mettre en application certaines directives médicales lorsqu'ils sont aux prises avec un problème de santé. Cette situation a clairement été identifiée au fil des ans, si bien que l'on reconnaît désormais l'importance de comprendre et de concilier les différentes perceptions du risque que ce soit de la part des publics, des experts, des gestionnaires ou de tout autre acteur clé (OMS, 2002; Massé, 1995).

Un exemple intéressant à cet égard est celui de l'hypertension artérielle (HTA). Ce problème préoccupant à l'échelle mondiale, tant par son ampleur que par sa gravité (OMS, 2002), a été associé, au fil des 
années, à la survenue d'accidents vasculaires cérébraux (AVC), à des coronaropathies, à des insuffisances cardiaques et rénales et à des maladies périphériques (Feldman et al., 1999; Campbell et Chockalingam, 1995 ; Frohlich, 1993 ; Joffres et al., 1992), ces maladies étant elles-mêmes responsables annuellement de près de $40 \%$ des décès chez les Canadiens et Canadiennes (FMCQ, 1999; Joffres et al., 1997). Or, si le risque cardiovasculaire associé à l'HTA et la pertinence du traitement médicamenteux sont bien établis aux yeux des experts, certains travaux ont montré qu'un même risque peut être interprété différemment par différents groupes au sein de la population (Gifford, 1986), ces interprétations relevant de plusieurs logiques ou rationalités contextualisées (Massé, 1995). Cet article a pour but d'illustrer les perceptions du risque chez des individus hypertendus considérés inobservants à leurs médicaments antihypertenseurs, puis d'analyser dans quelle mesure ces perceptions influent sur la prise irrégulière du médicament antihypertenseur. Nous mettons en évidence certains des enjeux relatifs au transfert des connaissances auprès des publics, auxquels les cliniciens sont appelés à porter un intérêt croissant.

\section{MÉTHODES}

Cette étude, de type qualitatif, a été menée entre 1998 et 2000 dans les régions de Montréal et de Québec auprès d'individus hypertendus considérés inobservants à leurs médicaments antihypertenseurs ${ }^{1}$. S'appuyant sur les principes méthodologiques de la théorisation ancrée (Glaser et Strauss, 1967), la démarche, essentiellement inductive, se refusait à toute construction à priori de concepts et d'hypothèses. La démarche impliquait un processus constant de questionnement jusqu'à l'atteinte d'une saturation sur le plan de l'articulation et des caractérisations théoriques et utilisait, pour ce faire, la procédure d'échantillonnage théorique (Laperrière, 1997 ; Paillé, 1994).

L'échantillon a donc été construit par vagues successives en fonction de sa pertinence théorique pour les fins de l'étude (Laperrière, 1997 ; Paillé, 1994). Nous avons d'abord cerné différentes variations ou manifestations de l'inobservance médicamenteuse parmi 22 hommes et femmes recrutés à travers une étude appelée Utilisation du médicament antihypertenseur (UMA). L'étude UMA a été menée entre les mois d'avril 1998 et mars 1999 par des chercheurs du Département de pharmacoépidémiologie de l'Université Laval à Québec et du Département de médecine familiale de l'Université McGill. Cette étude canadienne multicentrique avait pour but d'identifier les déterminants de l'inobservance de la prise de nouveaux médicaments antihypertenseurs chez des individus atteints d'HTA âgés de 17 ans et plus (Grégoire et al., 1995). Nos participants étaient recrutés par les chercheurs de l'étude UMA parmi les sujets qu'ils estimaient inobservants à partir d'un score obtenu à une échelle auto-administrée (Grégoire et al., 1992). Après avoir fait le bilan des différentes manifestations de l'inobservance médicamenteuse chez ces 22 participants (omission occasionnelle, report, cessation définitive, etc.) et ayant épuisé les possibilités de recrutement par l'étude UMA, nous avons, en dernier lieu, demandé à des médecins établis en clinique privée et en CLSC de nous fournir des sujets qui éprouvaient des difficultés importantes dans la prise de leur médicament antihypertenseur. Cinq autres participants ont été recrutés.

L'échantillon définitif de 27 participants couvre une diversité de manifestations de l'inobservance médicamenteuse soit l'omission occasionnelle de la prise du médicament, son report, l'omission de doses successives du médicament, la prise partielle du médicament (prise d'un médicament plutôt que deux, prise d'une dose plutôt que deux), la cessation temporaire et définitive de la prise du médicament. Quinze femmes et douze hommes âgés de

\section{TABLEAU 1 - CARACTÉRISTIQUES SOCIODÉMOGRAPHIQUES DES PARTICIPANTS}

\begin{tabular}{lr}
\hline Âge & N \\
\hline $40-54$ ans & 8 \\
$55-64$ ans & 11 \\
65 ans et plus & 8
\end{tabular}

\section{Sexe}

Femmes

15

Hommes

12

Formation

N'a pas complété ses études primaires

Niveau primaire complété

N'a pas complété ses études secondaires

Niveau secondaire complété

Niveau collégial complété

Niveau universitaire complété

\section{Occupation}

Salariés-es

Travailleurs et travailleuses autonomes

Retraités-es

Chômeurs et chômeuses

Assistés-es sociaux-ales

Accidentés-es du travail

$\mathrm{Au}$ foyer

\section{Lieux de résidence}

Milieu urbain

Banlieue

Milieu rural

\section{6}

3

2

3

5

8

8

(a)

6

3

10

2

1

1

4

40 à 70 ans composent cet échantillon. Ceux-ci proviennent de milieux urbains, semi-urbains et ruraux. Ils ont des niveaux de formation et des occupations variés (tableau 1). Leur durée d'utilisation d'un médicament antihypertenseur varie de un an à 37 ans.

Ces participants se sont prêtés à une entrevue en profondeur d'une durée approximative de 90 minutes. Dans le cadre de l'entrevue, nous avons couvert leurs perceptions, leurs attitudes, leurs connaissances et leurs actions relativement à l'HTA et au médicament antihypertenseur. S'y joignait une question centrale à l'étude visant à cerner leur prise irrégulière du médicament antihypertenseur. Nous leur demandions alors de nous parler de leurs difficultés relatives à la prise du médicament, tout en cherchant à connaître la manière dont ils justifiaient ces difficultés.

L'analyse des données a été effectuée en trois phases. La première a consisté à organiser le matériel par le biais de différentes opérations de codage, à le regrouper autour de catégories et à l'indexer à l'aide du logiciel QSR NUDIST. Nous avons ensuite procédé à des analyses ciblées ayant comme but d'explorer les logiques explicatives de la prise irrégulière du médicament antihypertenseur chez les 27 participants, et avons enfin cerné les régularités, les points de convergence et de divergence entre les différentes logiques personnelles d'inobservance afin d'en dégager les principales pistes d'analyse et de conclusion.

\section{RÉSULTATS}

À partir de l'exploration des logiques explicatives de la prise irrégulière du médicament antihypertenseur chez l'ensemble des participants, il a été possible de puiser différentes conceptions du risque associé à l'HTA ou au médicament antihypertenseur et conséquemment, d'identifier des façons distinctes de gérer le risque.

D'abord, plusieurs participants, généralement assidus dans la prise de leur médicament antihypertenseur, associent clairement un risque à la maladie. Les conséquences de l'HTA non contrôlée sont en effet bien réelles dans l'esprit de ces participants. Un interviewé dépeint avec force ses peurs, concevant que l'HTA puisse provoquer un décès foudroyant, soit une thrombose, en affectant le cœur après l'éclatement des veines. D'autres ont particulièrement peur de la maladie du fait que leur pression artérielle était et se maintient à un niveau élevé ou est difficile à stabiliser. Certains dépeignent les moments d'angoisse qu'ils ont vécus au moment où ils ont été hospitalisés à cause du niveau anormalement élevé de leur pression artérielle. Élément significatif, plusieurs de ces parti- 


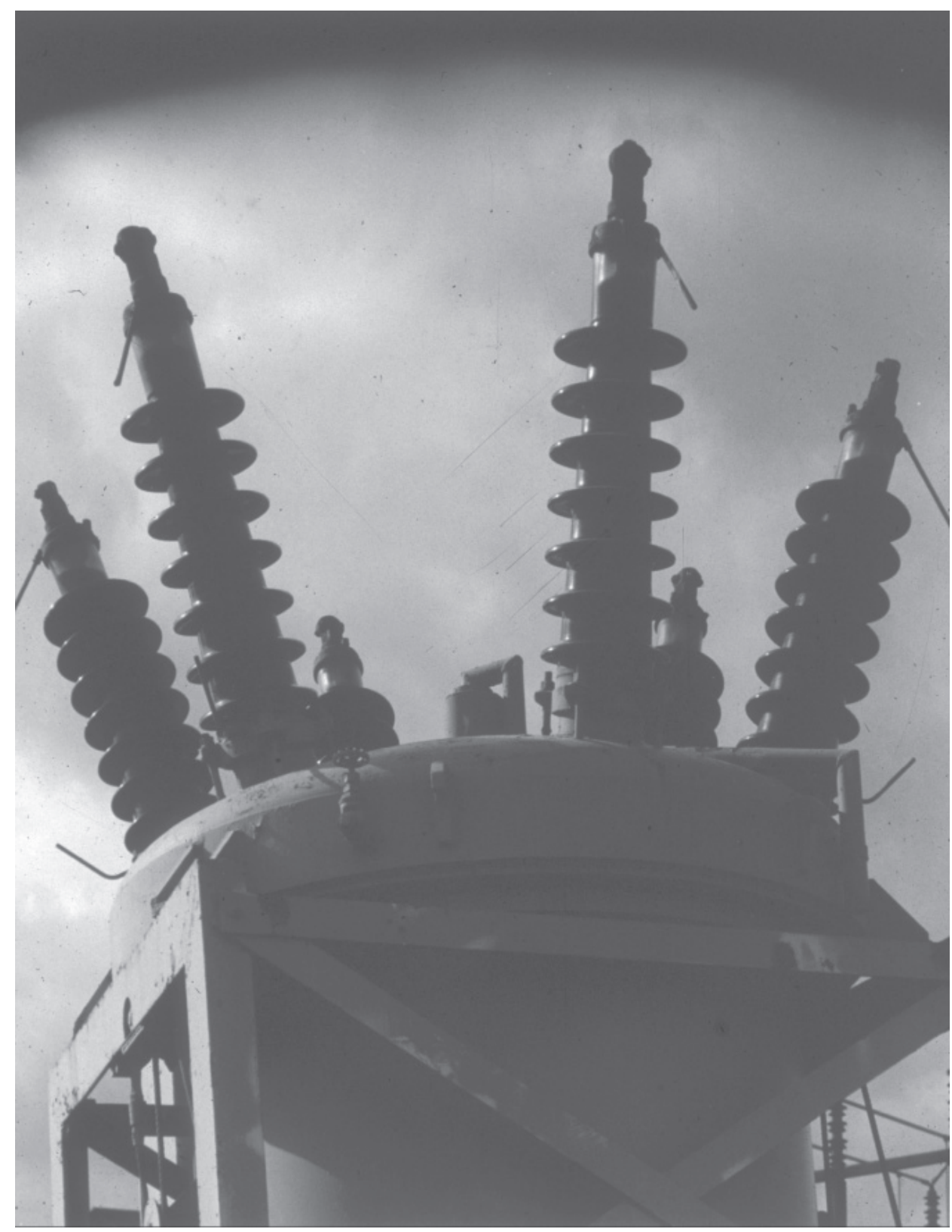

cipants ont été témoins des conséquences graves de l'HTA non contrôlée chez leurs proches (un des deux parents, frères, oncles) et d'autres observent ces mêmes conséquences dans leur entourage (voisins). La plupart du temps, ces personnes sont décédées, ont été victimes d'un accident cardiovasculaire (AVC) ou ont été paralysées des suites d'une HTA non contrôlée, parce qu'elles avaient cessé de prendre leur médicament, le prenaient de façon irrégulière ou l'avaient remplacé par des produits naturels. Ainsi, il est clair ici que le risque associé à la maladie prend une forme tangible dans l'expérience de ces participants. C'est d'ailleurs ce qui les motive à privilégier la prise régulière du médicament antihypertenseur. Ceux-ci se disent rassurés par le médicament, lui attribuant une fonction symbolique de protection, en plus de ont commencé à le prendre. La nature asymptomatique de l'HTA et, conséquemment, l'absence d'un risque que la maladie représentait, est alors un élément d'explications évoqué par ceux-ci. Le fait qu'ils se sentaient bien et que leur pression artérielle était stabilisée les a initialement incités à cesser temporairement de le prendre ou à omettre de prendre des doses successives du médicament. L'idée générale est la suivante : certains de ces participants se percevaient rétablis compte tenu que leur pression artérielle était stabilisée. D'autres, parce qu'ils se sentaient bien, ne se considéraient pas suffisamment atteints et ainsi ne trouvaient pas vraiment justifiée la prise du médicament antihypertenseur. En somme, il serait ici possible de dire que la prise irrégulière du médicament antihypertenseur chez ces individus répond à une phase initiale d'ajustement face à un risque mitigé qu'ils associaient à la maladie. La représentation que ces individus se font de leur risque évolue par contre dans le temps, ce qui les conduit, au moment de l'entrevue, à être généralement assidus dans la prise de leur médicament antihypertenseur.

Nous observons enfin un certain déplacement dans la conceptualisation que d'autres participants se font de leur risque, lequel est chez eux fortement incarné par le médicament antihypertenseur plutôt que par l'hypertension artérielle elle-même. En effet, ces participants se disent particulièrement réticents à prendre un médicament quel qu'il soit. Ils n'aiment pas, notent-ils, l'idée d'ingérer un produit chimique sur une base quotidienne et de surcroît à vie et considèrent qu'il y a un risque, à long terme, de causer du tort à l'organisme. Certains se disent nettement antimédicament, l'associant à une certaine servitude. D'autres encore craignent l'effet toxique du médicament et disent préférer ou avoir tendance à se tourner vers les approches naturelles de soins. Il semble alors que le médicament soit conceptualisé comme un produit synthétique, en contraste avec certaines thérapies alternatives qui leur paraissent beaucoup plus naturelles. Élément significatif, l'estimation que ces individus font de leur risque à prendre un médicament est généralement basée sur leurs expériences personnelles ou celles vécues par les proches. Une participante conçoit qu'elle a toujours pris une trop grande quantité de médicaments et $\mathrm{y}$ associe un risque pour sa santé. Elle se sent ainsi justifiée de ne prendre qu'une dose partielle de son médicament antihypertenseur, refusant catégoriquement de prendre deux médicaments par jour, malgré les mises en garde de son médecin. Chez d'autres, la persistance d'effets secondaires avec les années paraît être déterminante. Une interviewée qui 
prend un médicament antihypertenseur depuis 27 ans se dit particulièrement hypothéquée par sa médication. Cette participante qui a toujours souffert d'effets secondaires au médicament a dû fréquemment changer de médicaments. Elle déplore, en plus, que les médicaments antihypertenseurs ne parviennent pas à stabiliser sa pression artérielle et pense, en définitive, que le médicament lui cause plus de désagréments que la maladie elle-même. Or, ces participants ont cela de particulier qu'ils persistent, au moment de l'entrevue, dans la prise irrégulière du médicament antihypertenseur et ce, même après quelques années de la prise du médicament. Seul l'un d'eux, qui prend un médicament antihypertenseur depuis 25 ans, précise que jusqu'à tout récemment, il ne prenait pas son médicament de façon régulière. Depuis quelques mois, la peur des conséquences de l'HTA le motive, note-t-il, à prendre régulièrement son médicament et ce, depuis qu'il côtoie des personnes ayant été victimes d'AVC.

\section{DISCUSSION}

Ces résultats permettent d'entrevoir la manière dont se construit le risque chez des individus hypertendus, la façon dont ils le gèrent et, conséquemment, la manière dont ils décident de prendre leur médicament antihypertenseur. Un certain nombre d'aspects sont alors portés à notre attention. D'abord, l'étude laisse apparaître à quel point la nature même de l'HTA a une certaine influence sur la façon dont certains individus ont initialement conceptualisé leur risque. Il est clair en effet qu'un certain nombre de participants associaient un risque mitigé, sinon nul à la maladie, du fait qu'ils se sentaient bien et qu'ils ne ressentaient aucun symptôme. Précisons, sur ce point, que l'HTA est généralement considérée comme une maladie asymptomatique. À l'exception d'individus atteints à des stades plus avancés (Williams, 1998; Isselbacher et al., 1980), la seule rétroaction que la plupart des individus ont face à leur état de santé prend une forme abstraite exprimée par des valeurs tensionnelles (Joffres et al., 2001) plutôt que par des signaux du corps (Kjellgren et al., 1997 ; Grueninger, 1995). L'HTA ne donne pas aux individus de signes ou des manifestations tangibles qui les motiveraient éventuellement à prendre un médicament antihypertenseur et de surcroît à vie (Allen, 1998). Les études indiquent bien, à ce sujet, que les individus atteints d'HTA sont généralement réticents à prendre un médicament antihypertenseur à vie, à plus forte raison lorsqu'ils ne ressentent aucun symptôme et lorsque le médicament ne donne pas les résultats attendus (Rehder et al., 1980 ; Geertsen et al., 1973). Or, l'étude

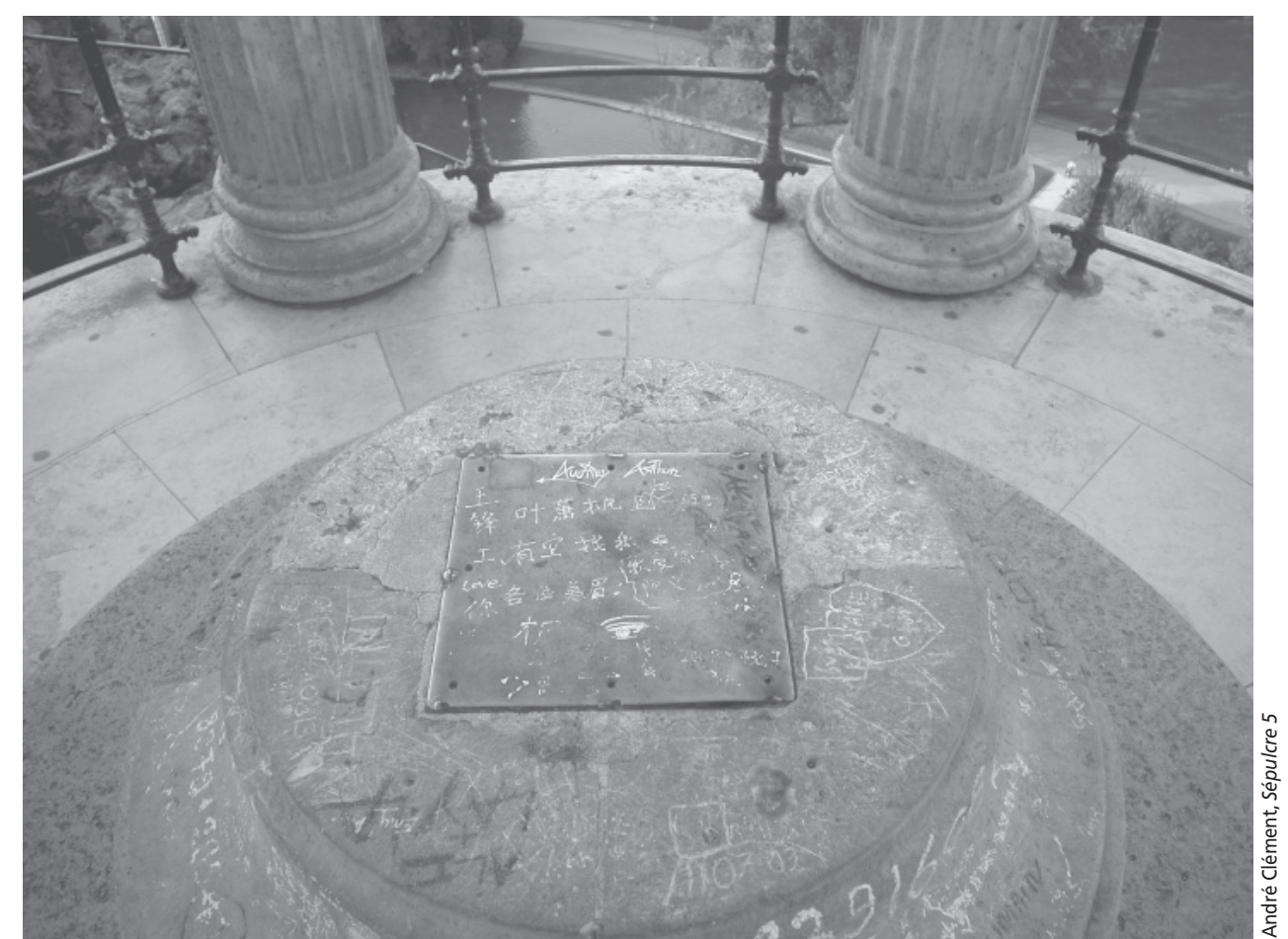

permet clairement de voir que l'acceptabilité du risque chez les participants et, conséquemment, du traitement proposé, est fonction de nombreux aspects qui relèvent tant de l'expérience personnelle que de l'observation d'expériences. En outre, l'étude permet de voir que le risque associé à la maladie prend une forme tangible dans l'expérience des participants, après qu'ils ont été témoins d'accidents graves chez leurs proches atteints de la maladie C'est nettement à travers l'observation de ces expériences que ces individus ont progressivement évalué leur propre risque face à la maladie (Kjellgren et al., 1997 ; Bury, 1994 ; Davison et al., 1992 ; Davison et al., 1991), ce qui les a motivés à prendre le plus régulièrement possible leur médicament antihypertenseur.

Par ailleurs, l'étude laisse apparaître un certain déplacement dans la conceptualisation du risque chez d'autres participants, lequel est alors nettement incarné par le médicament. Le médicament prend ici une forme menaçante, risquant de causer des dommages importants et irréversibles à l'organisme et ce, en raison de son caractère dénaturé. Ces représentations associées au médicament ont été abondamment décrites dans les écrits (Morgan, 1996; Britten, 1994, 1996; Fallsberg, 1991; Morgan et Watkins, 1988; Aballea, 1987). En effet, bon nombre d'études mettent au jour la méfiance que des individus entretiennent à l'égard du médicament (Britten, 1996). Tout comme le souligne notre étude, diverses recherches indiquaient déjà que certains individus n'aiment pas l'idée d'ingérer quo- tidiennement un produit chimique, leur vie durant, craignant à long terme, de causer du tort à l'organisme (Britten, 1994 ; Morgan et Watkins, 1988 ; Aballea, 1987). Certains associent le médicament à une certaine servitude (Morgan, 1996; Britten, 1994, 1996) ou à du poison, soulignant alors l'effet potentiellement toxique du médicament (Fallsberg, 1991; Aballea, 1987). Or, au-delà de ces représentations du risque, les expériences personnelles de même que l'observation et la discussion de cas dans le réseau personnel paraissent aussi conforter ces participants à propos du risque que représente le médicament (Hunt et Emslie, 2001 ; Lupton, 1995 ; Backett et al., 1994; Kelleher et al., 1994). Chez certains de ces participants, c'est la persistance avec les années d'effets secondaires qui paraît donner une certaine matérialité au risque. Cet aspect est d'ailleurs retenu dans les écrits comme dimension explicative de l'inobservance aux médicaments antihypertenseurs (Kjellgren et al., 1997 ; Rudd, 1995 ; Morris et Schultz, 1993 ; Richardson et al., 1993 ; Fallsberg, 1991). La quantité de médicaments à prendre paraît aussi intervenir chez une participante âgée qui se sent alors justifiée de prendre moins de médicaments que recommandé. Soulignons ici le caractère dissonant de la situation dans laquelle se trouve cette participante. Les médias et les scientifiques ont abondamment traité, ces dernières années, de nombreuses controverses entourant la surconsommation de médicaments, particulièrement chez les personnes âgées (Santé Québec, 1987, 1995). Il est possible ici de 
penser que cette participante soit sensible à ces controverses. On peut dès lors comprendre qu'elle puisse hésiter à prendre sa médication comme recommandée, devant une information ambiante qui met bien en évidence la menace que représente la prise de médicaments en grandes quantités. Or, indépendamment du caractère dissonant de la situation, il est clair que le seul fait de prendre une grande quantité de médicaments peut poser des difficultés et influer sur la prise d'un médicament quel qu'il soit. La complexité du régime thérapeutique, soit le nombre de médicaments consommés quotidiennement et l'administration du même médicament en plusieurs doses quotidiennes, est d'ailleurs retenue par maints auteurs comme un déterminant de l'inobservance aux traitements (Allen, 1998; Chockalingam et al., 1998 ; Feldman et al., 1998 ; Yasin, 1998 ; Leenen et al., 1997 ; Mancia, 1995 ; Sanson-Fisher et Clover, 1995 ; Klein, 1988; Morgan et al., 1986; Conrad, 1985 ; Luscher et Vetter, 1982). En définitive, tant les représentations du médicament et les controverses qui demeurent présentes au sujet de la prise de médicaments, que les expériences personnelles et celles des proches paraissent rendre particulièrement difficile un certain alignement de ces individus avec ce qui est recommandé.

\section{LE CHAMP DE LA PERCEPTION DU RISQUE COMME AVENUE PROMETTEUSE POUR LE TRANSFERT DES CONNAISSANCES}

Le champ d'études s'intéressant à la perception du risque est encore peu documenté et articulé dans les écrits (OMS, 2002). Il est pourtant une avenue de recherche prometteuse en médecine clinique, non seulement, pour mieux comprendre les prises irrégulières de médicaments chez les individus atteints, mais aussi les aspects essentiels au transfert des connaissances du point de vue des publics. En effet, le transfert des connaissances paraît vain, s'il ne conduit pas à de nouvelles façons de faire auprès d'individus ou des populations concernés, en prenant en compte particulièrement leur propre perspective et conceptions du risque. L'analyse des perceptions du risque chez différents groupes de la population, dans différents contextes et pour des pathologies différentes, paraît ainsi fondamentale pour que devienne possible un certain arrimage entre les modèles explicatifs de la maladie chez les individus atteints et les professionnels de la santé. Également, de façon à faciliter cet arrimage, les experts et les professionnels de la santé devraient donner accès aux individus atteints et même à la population en général, à une information vulgarisée sur les risques, la plus complète et la plus transparente possible, soit une information qui présenterait clairement et dans un langage accessible, les controverses, les certitudes et les incertitudes entourant une pathologie donnée et le médicament lui-même. Le transfert des connaissances devrait enfin se faire dans le cadre d'un dialogue franc et ouvert entre le professionnel de la santé et son patient.

\section{Bibliographie}

ABALLEA, F. (1987). Le besoin de santé, les déterminants sociaux de la consommation, France, Presses universitaires de France.

ALLEN, H. (1998). «Promoting compliance with antihypertensive medication », British Journal of Nursing, vol. 7, $\mathrm{n}^{\circ}$ 20, p. 1252-1258.

BACKETT, K., C. DAVISON et K. MULLEN (1994). "Lay evaluation of health and healthy lifestyles: Evidence from three studies", British Journal of General Practice, vol. 44, $\mathrm{n}^{\circ} 383$, p. 277-280.

BRITTEN, N. (1994). «Patients' ideas about medicines: A qualitative study in a general practice population», British Journal of General Practice, vol. 44, p. 465-468.

BRITTEN, N. (1996). «Lay views of drugs and medicines: Orthodox and unorthodox accounts, dans S.J. WILLIAMS et $\mathrm{M}$. CALNAN, Modern medicine: Lay perspectives and experiences, Londres, UCL Press Ltd., p. 48-73.

BURY, M. (1994). "Health promotion and lay epidemiology: A sociological view», Health Care Analysis, no 2, p. 23-30.

CAMPBELL, N.R. et A. CHOCKALINGAM (1995). «Prevention and control of high BP : Challenges and opportunities », Canadian Medical Association Journal, vol. 152, $\mathrm{n}^{\circ}$ 12, p. 1969-1970.

CHOCALINGAM, A., M. BACHER, N. CAMPBELL, H. CUTLER, A. DROVER, FELDMAN et al. (1998). "Adherence to management of high blood pressure: Recommandations of the Canadian Coalition for High Blood Pressure Prevention and Control ", Canadian Journal of Public Health, vol. 89, n 5 , I-5-I-7.

CONRAD, P. (1985). "The meaning of medications : Another look at compliance», Social Science and Medicine, vol. 20, $\mathrm{n}^{\circ}$ 1, p. 29-37.

DAVISON, C., G. DAVEY SMITH, et S. FRANKEL (1991). "Lay epidemiology and the prevention paradox: The implications of coronary candidacy for health education ", Sociology of Health and Illness, vol. 13, n 1, p. 1-19.

DAVISON, C., S. FRANKEL, et G.D. SMITH (1992). "To hell with tomorrow: Coronary heart disease risk and the ethnography of fatalism ", dans S. SCOTT, G. WILLIAMS, S. PLATT et H. THOMAS (dir.), Private Risks and Public Dangers. Exploration in Sociology, Aldershot, Brookfield, É.-U., Hong Kong, Singapore, Sydney, Avebury, no 43, p. 95-111.

DUPUIS, J.P. et S. KARSENTY (1974). L'invasion pharmaceutique, Paris, Éditions du Seuil.
FALLSBERG, M. (1991). Reflections on medicines and medication: A qualitative analysis among people on long-term drug regimens, thèse de doctorat, Department of Education and Psychology (Dissertations $\mathrm{n}^{\circ}$ 31), Suède, Linkoping University.

FELDMAN, R.D., N.R.C. CAMPBELL et P. LAROCHELLE (1999). "Clinical problem solving based on the 1999. Canadian recommendations for the management of hypertension », Canadian Medical Association Journal, vol. 161, n 12, SF21-SF25.

FELDMAN, R., M. BACHER, N. CAMPBELL, A. DROVER et A. CHOCKALINGAM (1998). "Adherence to pharmacologic management of hypertension », Canadian Journal of Public Health, n ${ }^{\circ} 89$, suppl. 1, p. 16-18.

FONDATION DES MALADIES DU CEEUR (1999). Le nouveau visage des maladies cardiovasculaires et des accidents vasculaires cérébraux au Canada 2000. Collection Laboratoire de lutte contre la maladie, Statistique Canada: Institut canadien d'information sur la santé, Société canadienne de cardiologie, Canadian Stroke Society.

FROLICH, E.D. (1993). «The JNC-V : Consensus recommendations for the treatment of hypertension », Drug Therapy, juin 1993, p. 23-56.

GEERTSEN, H.R., R.M. GRAY et J.R. WARD (1973). «Patient non-compliance within the context of seeking medical care for arthritis », Journal of Chronic Disease, vol. 26, p. 689698.

GIFFORD, S. (1986). "The meaning of lumps: A case study of the ambiguities of risk ", dans R. STALL, C. JAMES et S. GIFFORD, Anthropology and epidemiology: interdisciplinary approaches to the study of health and disease, Dordrecht (Pays-Bas), Reidel Publishing, p. 213-246.

GLASER, B.G. et A.L. STRAUSS (1967). The discovery of grounded theory. Strategies for qualitative research, Chicago, Aldine.

GRÉGOIRE, J.-P., R. GUIBERT, A. ARCHAMBAULT et A.-P. CONTANDRIOPOULOS (1992). "Medication compliance in a family practice», Canadian Family Physician, $\mathrm{n}^{\circ}$ 38, p. 2333-2337.

GRÉGOIRE, J.-P., J. MOISAN, R. GUIBERT, A. CIAMPI et A. MILOT (1995). Use of medication in hypertension (UMH): The impact of side effects on compliance to drug treatment, manuscrit d'un protocole de recherche, Unité de pharmacoépidémiologie et Département de médecine de l'Université Laval à Québec, Département de médecine familiale et d'épidémiologie et biostatistique de l'Université McGill.

GRUENINGER, U.J. (1995). «Arterial hypertension: Lessons from patient education» Patient Education and Counselling, $\mathrm{n}^{\circ} 26$, p. 37-55.

HUNT, K. et C. EMSLIE (2001). «Commentary: The prevention paradox in lay epidemiology- Rose revisited », International Journal of Epidemiology, $\mathrm{n}^{\circ} 30$, p. 442446. 
ISSELBACHER, K.T., R.D. ADAMS, E. BRAWNWALD, R.G. PETERSDORF et J.D. WILSON (1980). Harrison's principles of internal medicine (9th ed.), New York, McGraw-Hill.

KELLEHER, D., J. GABE et G. WILLIAMS (1994). «Understanding medical dominance in the modern world", dans J. GABE, D. KELLEHER et G. WILLIAMS, Challenging Medicine, Londres et New York, Routledge, p. XXI-XXIX.

KJELLGREN, K.I., S. SVENSSON, J. AHLNER et R. SALJO (1997). "Hypertensive patients' knowledge of high blood pressure », Scandinavian Journal of Primary Health Care, vol. 15, n 4, p. 188-192.

KLEIN, L.E. (1988). "Compliance and blood pressure control», Hypertension, vol. 11 (3 Pt. 2), p. 1161-1164.

JOFFRES, M.R., P. HAMET, S.W. RABKIN, D. GELSKEY, K. HOGAN et G. FODOR (1992). "Prevalence, control and awareness of high blood pressure among Canadian adults», Canadian Medical Association, vol. 146, n 11 , p. 1997-2005.

JOFFRES, M.R., P. GHADIRIAN, G. FODOR, A. PETRASOVITS, A. CHOCKALINGAM et P. HAMET (1997). "Awareness, treatment and control of hypertension in Canada", American Journal of Hypertension, $\mathrm{n}^{\circ} 10$, p. 1097-1102.

JOFFRES, M.R., P. HAMET, D.R. MCLEAN, G.J. L'ITALIEN et G. FODOR (2001). "Distribution of blood pressure and hypertension in Canada and the United States", American Journal of Hypertension, $\mathrm{n}^{\circ} 14$, p. 1099-1105.

LAPERRIÈRE, A. (1997). «La théorisation ancrée (grounded theory): démarche analytique et comparaison avec d'autres approches apparentées », dans J. POUPART, L.-H. GROULX, J.-P. DESLAURIERS, A. LAPERRIÈRE, R. MAYER, et A.P. PIRES. La recherche qualitative: enjeux épistémologiques et méthodologiques, Montréal, Paris, Casablanca, Gaëtan Morin Éditeur, p. 309-333.

LEENEN, F.H.H., T.W. WILSON, P. BOLLI, P. LAROCHELLE, M. MYERS, S.P. HANDA et al. (1997). "Patterns of compliance with once versus twice daily antihypertensive drug therapy in primary care: A randomized clinical trial using electronic monitoring ", Canadian Journal of Cardiology, vol. 13, n 10 , p. 914-1159.

LUSCHER, T.F. et W. VETTER (1982). "Adherence to medication", Journal of Human Hypertension, vol. 1, $\mathrm{n}^{\circ} 4$ (suppl.), p. 43-46.

LUPTON, D. (1995). The imperative of health: Public health and regulated body, Londres, Thousands Oaks, New Delhi, Sage Publications.

MANCIA, G. (1995). "Improving the management of hypertension in clinical practice», Journal of Human Hypertension, $\mathrm{n}^{\circ}$ 9, S29-31.

MASSÉ, R. (1995). Culture et santé publique: les contributions de l'anthropologie à la prévention et la promotion de la santé, Montréal, Paris, Casablanca, Gaëtan Morin Éditeur.

MONTAGNE, M. (1992). «The promotion of medication for personal and social problems", The Journal of Drug Issues, vol. $22, \mathrm{n}^{\circ} 2$, p. 389-405.

MORGAN, M. et C.J. WATKINS (1988). «Managing hypertension: Beliefs and responses to medication among cultural groups ", Sociology of Health and Illness, $\mathrm{n}^{\circ} 10$, p. 561-577.

MORGAN, M. (1996). «Perceptions and use of antihypertensive drugs among cultural groups ", dans S.J. WILLIAMS et M. CALNAN, Modern medicine: Lay perspectives and experiences, Londres, UCL Press, p. 95-115.

MORGAN, T.O., C. NOWSON, J. MURPHY et R. SNOWDEN (1986). "Compliance and the elderly hypertensive », Drugs, $\mathrm{n}^{\circ} 31$ (Suppl. 4), p. 174-183.

MORRIS, S.L. et R.M. SCHULTZ (1993). "Medication compliance: The patients's perspective », Clinical Therapeutics, vol. 15, no 3, p. 593-606.

ORGANISATION MONDIALE DE LA SANTÉ (2002). "Rapport sur la santé dans le monde 2002: réduire les risques et pro- mouvoir une vie saine», rapport d'un comité d'experts, Genève (Série de rapports techniques, No. 2242002).

PAILLÉ, P. (1994). «L'analyse par théorisation ancrée", Cahiers de recherche sociologique, $\mathrm{n}^{\circ} 23$, p. 147-181.

REHDER, T.L., L.K. MCCOY, B. BLACKWELL, W. WHITEHEAD et A. ROBINSON (1980). «Improving medication compliance by counseling and special prescription container », American Journal of Hospital Pharmacy, ${ }^{\circ}$ 37, p. 379-385.

RICHARDSON, M.A., B. SIMONSMORTON et J.F. ANNEGERS (1993). « Effect of perceived barriers on compliance with antihypertensive medication », Health Education Quarterly, vol. 20, $\mathrm{n}^{\circ} 4$, p. 489-503.

RUDD, P. (1995). «Clinicians and patients with hypertension: Unsettled issues about compliance», American Heart Journal, vol. $130, n^{\circ} 3$, p. 572-579.

SANSON-FISCHER, R.W. et K. CLOVER (1995). "Compliance in the treatment of hypertension. A need for action ", American Journal of Hypertension, $\mathrm{n}^{\circ} 8$ (10 Pt 2), 82S-88S.

SANTÉ QUÉBEC (1987). Et la santé, ça va? Rapport de l'Enquête Santé Québec, tome 1, Ministère de la Santé et des Services sociaux, Québec, Les Publications du Québec.

SANTÉ QUÉBEC (1995). Et la santé, ça va en 1992-1993? Rapport de l'Enquête sociale et de santé 1992-1993, vol. 1, Montréal, Ministère de la Santé et des Services sociaux, Gouvernement du Québec.

WILLIAMS, D.R. (1998). «Assessing patient wellness: New perspective on quality of life and compliance», American Journal of Hypertension, $\mathrm{n}^{\circ} 11$ (11Pt 2), 186S-191S.

YASIN, S. (1998). «Detecting and improving compliance: Is concordance the solution?", Australian Family Physician, vol. 27, n ${ }^{\circ} 4$, p. $255-260$.

\section{Note}

1. Cette étude a été financée par le Conseil des recherches médicales du Canada (CRM). 